\title{
New numerical analysis of Riemann-Liouville time-fractional Schrödinger with power, exponential decay, and Mittag-Leffler laws
}

\author{
Badr Saad T. Alkahtani ${ }^{\mathrm{a}}$, Ilknur Koca ${ }^{\mathrm{b}}$, Abdon Atangana ${ }^{\mathrm{c}, *}$ \\ ${ }^{a}$ Department of mathematics, colle of science, King Saud University, P. O. Box 1142, Riyadh 11989, Saudi Arabia. \\ ${ }^{b}$ Department of Mathematics, Faculty of Sciences, Mehmet Akif Ersoy University, 15100, Burdur, Turkey. \\ ${ }^{c}$ Institute for Groundwater Studies, Faculty of Natural and Agricultural Sciences, University of the Free State, 9300, Bloemfontein, South \\ Africa. \\ Communicated by X.-J. Yang
}

\begin{abstract}
The mathematical equation that describes how the quantum state of a quantum system changes during time was considered within the framework of fractional differentiation with three different derivatives in Riemann-Liouville sense. The fractional derivatives used in this work are constructed based on power, exponential decay, and Mittag-Leffler law. A new numerical scheme for fractional derivative in Riemann-Liouville sense is presented and used to solve numerically the Schrödinger equation. The stability analysis of each model is presented in detail. (C)2017 All rights reserved.
\end{abstract}

Keywords: Power law, exponential decay law, Mittag-Leffler law, numerical scheme, Schrödinger equation. 2010 MSC: 26A33, 33E12, 35J10.

\section{Introduction}

The Riemann-Liouville fractional integral was obtained via the Cauchy formula for repeated integral $[7,10,11,17,18]$. The Riemann-Liouville fractional integral possesses important properties like the semigroup; the operator is indeed well-defined on the set of locally integrable function on the entire real line R. Using the fundamental relations of the Riemann-Liouville fractional integral, the Riemann-Liouville fractional derivative was constructed by taking enough derivative of the fractional integral. Nevertheless the Riemann-Liouville fractional derivative appears to be the derivative of a convolution of the power law $x^{-\alpha}$ with any continuous function $f$. This is a very interesting and important result because many natural occurrences seem to follow the power law. Nonetheless the utility of this power law in describing real world problem, the fractional derivative with kernel as power law has a problem of singularity at the origin, in addition to this; many other physical occurrences do not follow the power law used in Riemann-Liouville fractional derivative but follow the law of exponential decay. To solve first the problem of singularity and also that of exponential decay law, a derivative based on exponential decay

\footnotetext{
${ }^{*}$ Corresponding author

Email addresses: balqahtani1@ksu.edu.sa (Badr Saad T. Alkahtani), ikoca@mehmetakif .edu.tr (Ilknur Koca), abdonatangana@yahoo.fr (Abdon Atangana)
}

doi:10.22436/jnsa.010.08.18 
law kernel was suggested by Caputo and Fabrizio $[3,8,9,12]$. However, there is an established rule in the scope of fractional differentiation that states that any fractional derivative must have a non-local kernel. With this established rule, it appears that the Caputo-Fabrizio derivative is not a fractional operator however it is very useful in describing real world problems that follow the exponential decay law. To solve the problem of singularity, non-locality of the kernel and also to have added the more generalized decay law, Abdon Atangana and Dumitru Baleanu suggested a fractional derivative with the generalized Mittag-Leffler function as kernel [1, 2, 4, 6]. The definition of fractional derivative based on the RiemannLiouville approach although very useful but it numerical approximation has not gain attention in the field of fractional differentiation. Recently Atangana and Gomez suggested the numerical approximation of fractional derivative from power law to the generalized Mittag-Leffler function. In this paper, we solve numerically the Schrödinger equation with power law, exponential decay law and the generalized Mittag-Leffler law as kernels of time fractional derivative.

\section{Fractional order derivatives in Riemann-Liouville sense}

In this section, we present the fractional order definitions with Riemann-Liouville senses.

Definition 2.1. Let $f$ be a function not necessarily differentiable, and $\alpha$ be a real number such that $0 \leqslant$ $\alpha \leqslant 1$, then the derivative with $\alpha$ order with power law is given as [13]

$$
{ }^{R L} D_{t}^{\alpha}[f(t)]=\frac{1}{\Gamma(1-\alpha)} \frac{d}{d t} \int_{0}^{t}(t-y)^{-\alpha} f(y) d y .
$$

Definition 2.2. Let $f \in H^{1}(a, b), b>a, \alpha \in[0,1]$ and not necessarily differentiable, then, the definition of the new fractional derivative (Atangana-Baleanu fractional derivative in Riemann-Liouville sense) is given as [4]

$$
{ }^{A B R} D_{t}^{\alpha}[f(t)]=\frac{B(\alpha)}{1-\alpha} \frac{d}{d t} \int_{a}^{t} f(y) E_{\alpha}\left[-\frac{\alpha}{1-\alpha}(t-y)^{\alpha}\right] d y .
$$

Definition 2.3. Let $f$ be a function not necessarily differentiable, and $\alpha$ be a real number such that $0 \leqslant$ $\alpha \leqslant 1$, then the derivative with $\alpha$ order with exponential decay-law is given as [15]

$$
{ }^{C F R} D_{t}^{\alpha}[f(t)]=\frac{M(\alpha)}{1-\alpha} \frac{d}{d t} \int_{a}^{t} f(y) \exp \left[-\frac{\alpha}{1-\alpha}(t-y)\right] d y,
$$

where $M(\alpha)$ and $B(\alpha)$ denote the normalization functions obeying $M(0)=M(1)=1$ and $B(0)=B(1)=1$, respectively.

\section{Numerical approximations and stability analysis of numerical schemes}

In this section we will consider the time dependent Schrödinger equation for one spatial dimension that is given as below:

$$
\frac{-h^{2}}{2 m} \frac{\partial^{2} s(x, t)}{\partial x^{2}}+u(x) s(x, t)=i h \frac{\partial s(x, t)}{\partial t} .
$$

This section is structured as follows, the numerical analysis of the Schrödinger equation with RiemannLiouville fractional derivative is first presented, this will be followed by the analysis of the model with Caputo-Fabrizio derivative in Riemann-Liouville sense and finally the model with Atangana-Baleanu fractional derivative in Riemann-Liouville sense. The numerical approximation used in this paper can be found in [5]. 
3.1. Caputo-Fabrizio approach with RL sense and stability analysis of numerical scheme

The time dependent Schrödinger equation with CFR (Caputo-Fabrizio with Riemann Liouville sense) operator is given by

$$
\text { ih }{ }_{0}^{C F R} D_{t}^{\alpha}[s(x, t)]=\frac{-h^{2}}{2 m} \frac{\partial^{2} s(x, t)}{\partial x^{2}}+u(x) s(x, t), 0 \leqslant \alpha \leqslant 1 .
$$

The numerical approximation of Caputo-Fabrizio with Riemann Liouville sense is obtained with steps as below [14-16]

$$
{ }_{0}^{C F R} D_{t}^{\alpha}[f(t)]=\frac{M(\alpha)}{1-\alpha} \frac{d}{d t} \int_{0}^{t} f(y) \exp \left[-\frac{\alpha}{1-\alpha}(t-y)\right] d y
$$

If we take

$$
C(t)=\frac{M(\alpha)}{1-\alpha} \int_{0}^{t} f(y) \exp \left[-\frac{\alpha}{1-\alpha}(t-y)\right] d y
$$

then we have

$$
{ }_{0}^{C F R} D_{t}^{\alpha}[f(t)]=\frac{d}{d t} C(t)=\frac{C\left(t_{j+1}\right)-C\left(t_{j}\right)}{\Delta t}
$$

So we have

$$
{ }_{0}^{C F R} D_{t}^{\alpha}[f(t)]=\frac{M(\alpha)}{\alpha \Delta t}\left\{\begin{array}{c}
\sum_{k=0}^{j} \frac{f\left(t_{k+1}\right)+f\left(t_{k}\right)}{2}\left(\exp \left[-\frac{\alpha}{1-\alpha}\left(t_{j+1}-t_{k+1}\right)\right]-\exp \left[-\frac{\alpha}{1-\alpha}\left(t_{j+1}-t_{k}\right)\right]\right) \\
-\sum_{k=0}^{j-1} \frac{f\left(t_{k+1}\right)+f\left(t_{k}\right)}{2}\left(\exp \left[-\frac{\alpha}{1-\alpha}\left(t_{j}-t_{k+1}\right)\right]-\exp \left[-\frac{\alpha}{1-\alpha}\left(t_{j}-t_{k}\right)\right]\right)
\end{array}\right\}+C_{\alpha, j, k \prime}
$$

where $C_{\alpha, j, k}$ is the remainder term given by equality below

$$
C_{\alpha, j, k}=\frac{M(\alpha)}{(1-\alpha) \Delta t}\left\{\begin{array}{l}
\sum_{k=0}^{j} \int_{t_{k}}^{t_{k+1}}\left\{f(y)-f\left(t_{k+1}\right)\right\}\left(\exp \left[-\frac{\alpha}{1-\alpha}\left(t_{j+1}-y\right)\right]\right) d y \\
-\sum_{k=0}^{j-1} \int_{t_{k}}^{t_{k+1}}\left\{f(y)-f\left(t_{k+1}\right)\right\}\left(\exp \left[-\frac{\alpha}{1-\alpha}\left(t_{j}-y\right)\right]\right) d y
\end{array}\right\}
$$

In the rest of part we will consider Schrödinger equation with CFR approximation.

$$
\text { ih } \begin{aligned}
& \frac{M(\alpha)}{\alpha \Delta t}\left(\sum_{k=0}^{j} \frac{s\left(t_{k+1}\right)+s\left(t_{k}\right)}{2} c_{j, k}^{\prime}-\sum_{k=0}^{j} \frac{s\left(t_{k+1}\right)+s\left(t_{k}\right)}{2} c_{j, k}^{\prime \prime}\right) \\
& =\frac{-h^{2}}{2 m}\left[\frac{\left(s_{i+1}^{j+1}-2 s_{i}^{j+1}+s_{i-1}^{j+1}\right)-\left(s_{i+1}^{j}-2 s_{i}^{j}+s_{i-1}^{j}\right)}{2(\Delta x)^{2}}\right]+u_{i}^{j}\left[\frac{s_{i}^{j+1}+s_{i}^{j}}{2}\right],
\end{aligned}
$$

where

$$
\begin{aligned}
c_{j, k}^{\prime} & =\left(\exp \left[-\frac{\alpha}{1-\alpha}\left(t_{j+1}-t_{k+1}\right)\right]-\exp \left[-\frac{\alpha}{1-\alpha}\left(t_{j+1}-t_{k}\right)\right]\right), \\
c_{j, k}^{\prime \prime} & =\left(\exp \left[-\frac{\alpha}{1-\alpha}\left(t_{j}-t_{k+1}\right)\right]-\exp \left[-\frac{\alpha}{1-\alpha}\left(t_{j}-t_{k}\right)\right]\right) .
\end{aligned}
$$

For simplicity, let we take

$$
d_{i}^{j}=i h \frac{M(\alpha)}{2 \alpha \Delta t}, e_{i}^{j}=\frac{-h^{2}}{4 m(\Delta x)^{2}}, f_{i}^{j}=\frac{u_{i}^{j}}{2} .
$$


Then we write (3.1) again,

$$
\begin{aligned}
& d_{i}^{j}\left(\sum_{k=0}^{j}\left(s\left(t_{k+1}\right)+s\left(t_{k}\right)\right) c_{j, k}^{\alpha}-\sum_{k=0}^{j-1}\left(s\left(t_{k+1}\right)+s\left(t_{k}\right)\right) c_{j, k}^{\prime \alpha}\right) \\
& \quad=e_{i}^{j}\left[\left(s_{i+1}^{j+1}-2 s_{i}^{j+1}+s_{i-1}^{j+1}\right)-\left(s_{i+1}^{j}-2 s_{i}^{j}+s_{i-1}^{j}\right)\right]+f_{i}^{j}\left[s_{i}^{j+1}+s_{i}^{j}\right], \\
& d_{i}^{j}\left(s_{i}^{j+1}+s_{i}^{j}\right) c_{j, k}^{\alpha}-d_{i}^{j}\left(s_{i}^{j}+s_{i}^{j-1}\right) c_{j, k}^{\prime \prime}+d_{i}^{j} \sum_{k=0}^{j-1}\left(s_{i}^{k+1}+s_{i}^{k}\right) c_{j, k}^{\alpha}-d_{i}^{j} \sum_{k=0}^{j-2}\left(s_{i}^{k+1}+s_{i}^{k}\right) c_{j, k}^{\alpha} \\
& \quad=e_{i}^{j}\left[\left(s_{i+1}^{j+1}-2 s_{i}^{j+1}+s_{i-1}^{j+1}\right)-\left(s_{i+1}^{j}-2 s_{i}^{j}+s_{i-1}^{j}\right)\right]+f_{i}^{j}\left[s_{i}^{j+1}+s_{i}^{j}\right] .
\end{aligned}
$$

Now we rearrange the equality (3.2) again below:

$$
\begin{aligned}
\left(d_{i}^{j} c_{j, k}^{\prime}+2 e_{i}^{j}-f_{i}^{j}\right) s_{i}^{j+1}= & \left(d_{i}^{j}\left(c_{j, k}^{\prime \prime}-c_{j, k}^{\alpha}\right)-2 e_{i}^{j}+f_{i}^{j}\right) s_{i}^{j} \\
& -d_{i}^{j} \sum_{k=0}^{j-1}\left(s_{i}^{k+1}+s_{i}^{k}\right) c_{j, k}^{\alpha}+d_{i}^{j} \sum_{k=0}^{j-2}\left(s_{i}^{k+1}+s_{i}^{k}\right) c_{j, k}^{\prime \prime} \\
& +e_{i}^{j}\left(s_{i+1}^{j+1}+s_{i-1}^{j+1}+s_{i+1}^{j}-2 s_{i}^{j}+s_{i-1}^{j}\right)+d_{i}^{j} s_{i}^{j-1} c_{j, k}^{\prime \prime} .
\end{aligned}
$$

Now we will present the stability analysis of the numerical scheme.

\subsubsection{Stability analysis of the numerical scheme}

To show the efficiency of the numerical scheme with the stability analysis, we assume that $g_{i}^{j}=s_{i}^{j}-p_{i}^{j}$, where $p_{i}^{j}$ is the approximate solution of the modified equation at the given point in time and space $\left(x_{i}, t_{j}\right)$, $(i=1,2, \ldots, N, j=1,2, \ldots, M)$, also the error for approximation is given as $g^{j}=\left[g_{1}^{j}, g_{2}^{j}, \ldots, g_{N}^{j}\right]$.

The error committed while solving the Schrödinger equation with CFR is given as:

$$
\begin{aligned}
\left(d_{i}^{j} c_{j, k}^{\prime}+2 e_{i}^{j}-f_{i}^{j}\right) g_{i}^{j+1}= & \left(d_{i}^{j}\left(c_{j, k}^{\prime \prime}-c_{j, k}^{\alpha}\right)-2 e_{i}^{j}+f_{i}^{j}\right) g_{i}^{j} \\
& -d_{i}^{j} \sum_{k=0}^{j-1}\left(g_{i}^{k+1}+g_{i}^{k}\right) c_{j, k}^{\alpha}+d_{i}^{j} \sum_{k=0}^{j-2}\left(g_{i}^{k+1}+g_{i}^{k}\right) c_{j, k}^{\prime \prime} \\
& +e_{i}^{j}\left(g_{i+1}^{j+1}+g_{i-1}^{j+1}+g_{i+1}^{j}-2 g_{i}^{j}+g_{i-1}^{j}\right)+d_{i}^{j} g_{i}^{j-1} c_{j, k}^{\alpha} .
\end{aligned}
$$

While studying the stability, we take forms as below:

$$
g_{m}(x, t)=\exp [a t] \exp \left[i k_{m} x\right]
$$

And,

$$
\begin{aligned}
g_{n}^{j} & =\exp [a t] \exp \left[i k_{m} x\right], & g_{n}^{j+1} & =\exp [a(t+\Delta t)] \exp \left[i k_{m} x\right], \\
g_{n+1}^{j} & =\exp [a t] \exp \left[i k_{m}(x+\Delta x)\right], & g_{n-1}^{j} & =\exp [a t] \exp \left[i k_{m}(x-\Delta x)\right], \\
g_{n+1}^{j+1} & =\exp [a(t+\Delta t)] \exp \left[i k_{m}(x+\Delta x)\right], & g_{n-1}^{j+1} & =\exp [a(t+\Delta t)] \exp \left[i k_{m}(x-\Delta x)\right], \\
g_{n-1}^{j-1} & =\exp [a(t-\Delta t)] \exp \left[i k_{m}(x-\Delta x)\right], & g_{n}^{j-1} & =\exp [a(t-\Delta t)] \exp \left[i k_{m}(x)\right],
\end{aligned}
$$


where $k_{m}=\frac{\pi m}{L}, m=1,2, \ldots, M=\frac{L}{\Delta x}$. Now put the aboves in equation (3.3), then we obtain

$$
\begin{aligned}
& \left(d_{i}^{j} c_{j, k}^{\prime}+2 e_{i}^{j}-f_{i}^{j}\right) \exp [a(t+\Delta t)] \exp \left[i k_{m} x\right] \\
& =\left(d_{i}^{j}\left(\begin{array}{c}
\prime \prime \\
c_{j, k}^{\alpha}-c_{j, k}^{\alpha}
\end{array}\right)-2 e_{i}^{j}+f_{i}^{j}\right) \exp [a t] \exp \left[i k_{m} x\right] \\
& \quad-d_{i}^{j} \sum_{k=0}^{j-1}\left(\exp [a(t+\Delta t)] \exp \left[i k_{m} x\right]+\exp [a(\Delta t)] \exp \left[i k_{m} x\right]\right) c_{j, k}^{\alpha} \\
& +d_{i}^{j} \sum_{k=0}^{j-2}\left(\exp [a(t+\Delta t)] \exp \left[i k_{m} x\right]+\exp [a(\Delta t)] \exp \left[i k_{m} x\right]\right) c_{j, k}^{\alpha} \\
& \quad+e_{i}^{j}\left(\begin{array}{l}
\quad \exp [a(t+\Delta t)] \exp \left[i k_{m}(x+\Delta x)\right] \\
\quad \exp [a t] \exp \left[i k_{m}(x+\Delta x)\right]+\exp [a t] \exp \left[i k_{m}(x-\Delta x)\right]
\end{array}\right) \\
& \quad+d_{i}^{j} \exp [a(t-\Delta t)] \exp \left[i k_{m} \Delta x\right] c_{j, k}^{\alpha} .
\end{aligned}
$$

Let do simplication with $\exp [a t] \exp \left[i k_{m} x\right]$ on both sides, then we obtain the following,

$$
\begin{aligned}
& \left(d_{i}^{j} c_{j, k}^{\prime}+2 e_{i}^{j}-f_{i}^{j}\right) \exp [a(\Delta t)] \\
& =\left(d_{i}^{j}\left(c_{j, k}^{\prime \prime}-c_{j, k}^{\alpha}\right)-2 e_{i}^{j}+f_{i}^{j}\right)-d_{i}^{j} \sum_{k=0}^{j-1}(\exp [a(\Delta t)]+1) c_{j, k}^{\alpha}+d_{i}^{j} \sum_{k=0}^{j-2}(\exp [a(\Delta t)]+1) c_{j, k}^{\prime \prime} \\
& +e_{i}^{j}\left(\begin{array}{c}
\exp [a(\Delta t)] \exp \left[i k_{m}(\Delta x)\right] \\
+\exp [a(\Delta t)] \exp \left[i k_{m}(-\Delta x)\right] \\
+\exp \left[i k_{m}(\Delta x)\right]+\exp \left[i k_{m}(-\Delta x)\right]
\end{array}\right)+d_{i}^{j} \exp [a(-\Delta t)] c_{j, k}^{\alpha} .
\end{aligned}
$$

Rearranging the above, we have

$$
\begin{aligned}
& \exp [a(\Delta t)]\left\{d_{i}^{j} c_{j, k}^{\prime}+2 e_{i}^{j}-f_{i}^{j}-d_{i}^{j} \cdot j\left(c_{j, k}^{\prime \prime}-c_{j, k}^{\prime}\right)-e_{i}^{j}\left(\exp \left[i k_{m}(\Delta x)\right]+\exp \left[i k_{m}(-\Delta x)\right]\right)\right\} \\
& \quad=\left\{\begin{array}{c}
d_{i}^{j}\left(c_{j, k}^{\prime \prime}-c_{j, k}^{\alpha}\right)-2 e_{i}^{j}+f_{i}^{j}+d_{i}^{j} \cdot j\left(c_{j, k}^{\prime \prime}-c_{j, k}^{\alpha}\right. \\
+e_{i}^{j}\left(\exp \left[i k_{m}(\Delta x)\right]+\exp \left[i k_{m}(-\Delta x)\right]\right)+d_{i}^{j} \exp [a(-\Delta t)] c_{j, k}^{\alpha}
\end{array}\right\} .
\end{aligned}
$$

Then

$$
\exp [a(\Delta t)]=\frac{\left\{\begin{array}{c}
d_{i}^{j}\left(c_{j, k}^{\prime \prime}-c_{j, k}^{\alpha}\right)-2 e_{i}^{j}+f_{i}^{j}+d_{i}^{j} \cdot j\left(c_{j, k}^{\prime \prime}-c_{j, k}^{\prime \alpha}\right) \\
+e_{i}^{j}\left(\exp \left[i k_{m}(\Delta x)\right]+\exp \left[i k_{m}(-\Delta x)\right]\right)+d_{i}^{j} \exp [a(-\Delta t)] c_{j, k}^{\alpha}
\end{array}\right\}}{\left\{d_{i}^{j} c_{j, k}^{\prime}+2 e_{i}^{j}-f_{i}^{j}-d_{i}^{j} \cdot j\left(c_{j, k}^{\prime \prime}-c_{j, k}^{\alpha}\right)-e_{i}^{j}\left(\exp \left[i k_{m}(\Delta x)\right]+\exp \left[i k_{m}(-\Delta x)\right]\right)\right\}} .
$$

Note that the condition for stability analyis is given by the following inequality

$$
\frac{g_{i}^{j+1}}{g_{i}^{j}}=\exp [a(\Delta t)] .
$$


Thus if

$$
\left|\frac{g_{i}^{j+1}}{g_{i}^{j}}\right| \leqslant 1,
$$

from equation (3.4)-(3.5) we have the following

$$
\left|\frac{g_{i}^{j+1}}{g_{i}^{j}}\right|=\exp [a(\Delta t)]|=| \frac{\left\{\begin{array}{c}
d_{i}^{j}\left(\begin{array}{c}
\prime \prime \\
\left.c_{j, k}^{\alpha}-c_{j, k}^{\alpha}\right) \\
+e_{i}^{j}\left(\exp \left[i k_{m}(\Delta x)\right]+\exp \left[i k_{m}^{j}(-\Delta x)\right]\right. \\
\prime \prime
\end{array} f_{i}^{j}+d_{i}^{j} \cdot j\left(\begin{array}{c}
\prime \prime \\
c_{j, k}^{\alpha}-c_{j, k}^{\alpha}
\end{array}\right)\right. \\
+d_{i}^{j} \exp [a(-\Delta t)] c_{j, k}^{\alpha}
\end{array}\right\}}{\left\{\begin{array}{c}
d_{i}^{j} c_{j, k}^{\prime}+2 e_{i}^{j}-f_{i}^{j}-d_{i}^{j} \cdot j\left(c_{j, k}^{\prime \prime}-c_{j, k}^{\alpha}\right) \\
-e_{i}^{j}\left(\exp \left[i k_{m}(\Delta x)\right]+\exp \left[i k_{m}(-\Delta x)\right]\right.
\end{array}\right\}} \mid
$$

Then the condition for stability is given as

$$
\left|\frac{\left\{\begin{array}{c}
d_{i}^{j}\left(\begin{array}{c}
\prime \prime \\
c_{j, k}^{\alpha}-c_{j, k}^{\prime}
\end{array}\right)-2 e_{i}^{j}+f_{i}^{j}+d_{i}^{j} \cdot j\left(\begin{array}{c}
\prime \prime \\
c_{j, k}^{\alpha}-c_{j, k}^{\alpha}
\end{array}\right) \\
+e_{i}^{j}\left(\exp \left[i k_{m}(\Delta x)\right]+\exp \left[i k_{m}(-\Delta x)\right]\right)+d_{i}^{j} \exp [a(-\Delta t)] c_{j, k}^{\alpha}
\end{array}\right\}}{\left\{d_{i}^{j} c_{j, k}^{\prime}+2 e_{i}^{j}-f_{i}^{j}-d_{i}^{j} \cdot j\left(c_{j, k}^{\prime \prime}-c_{j, k}^{\alpha}\right)-e_{i}^{j}\left(\exp \left[i k_{m}(\Delta x)\right]+\exp \left[i k_{m}(-\Delta x)\right]\right)\right\}}\right| \leqslant 1 .
$$

Thus from the above statement we can present the following theorem.

Theorem 3.1. The Crank-Nicholson scheme for solving the fractional Schrödinger equation with CFR is stable if inequality (3.6) is satisfied.

\subsection{Riemann-Liouville approach and stability analysis of numerical scheme}

The time dependent Schrödinger equation with RL (Riemann Liouville sense) operator is given by

$$
\text { ih }{ }_{0}^{R L} D_{t}^{\alpha}[s(x, t)]=\frac{-h^{2}}{2 m} \frac{\partial^{2} s(x, t)}{\partial x^{2}}+u(x) s(x, t), 0 \leqslant \alpha \leqslant 1 .
$$

The numerical approximation Riemann Liouville derivative is given below.

$$
{ }_{0}^{R L} D_{t}^{\alpha}[f(t)]=\frac{1}{\Gamma(1-\alpha)} \frac{d}{d t} \int_{0}^{t}(t-y)^{-\alpha} f(y) d y, 0 \leqslant \alpha \leqslant 1 .
$$

If we do same steps like numerical approximation of CFR, we will obtain below:

$$
{ }_{0}^{R L} D_{t}^{\alpha}[f(t)]=\frac{1}{\Gamma(2-\alpha) \Delta t}\left\{\begin{array}{c}
\sum_{k=0}^{j} \frac{f\left(t_{k+1}\right)+f\left(t_{k}\right)}{2}\left(\left(t_{j+1}-t_{k}\right)^{1-\alpha}-\left(t_{j+1}-t_{k+1}\right)^{1-\alpha}\right) \\
-\sum_{k=0}^{j-1} \frac{f\left(t_{k+1}\right)+f\left(t_{k}\right)}{2}\left(\left(t_{j}-t_{k}\right)^{1-\alpha}-\left(t_{j}-t_{k+1}\right)^{1-\alpha}\right)
\end{array}\right\}+R_{\alpha, j, k}
$$


where $R_{\alpha, j, k}$ is the remainder term given by equality

$$
R_{\alpha, j, k}=\frac{1}{(1-\alpha) \Delta t}\left\{\begin{array}{l}
\sum_{k=0}^{j} \int_{t_{k}}^{t_{k+1}}\left\{f(y)-f\left(t_{k+1}\right)\right\}\left(t_{j+1}-y\right)^{-\alpha} d y \\
-\sum_{k=0}^{j-1} \int_{t_{k}}^{t_{k+1}}\left\{f(y)-f\left(t_{k+1}\right)\right\}\left(t_{j}-y\right)^{-\alpha} d y
\end{array}\right\} .
$$

Now we will consider the Schrödinger equation with RL sense approximation.

$$
\text { ih } \begin{aligned}
& \frac{1}{\Gamma(2-\alpha) \Delta t}\left(\sum_{k=0}^{j} \frac{s\left(t_{k+1}\right)+s\left(t_{k}\right)}{2} r_{j, k}^{\prime}-\sum_{k=0}^{j-1} \frac{s\left(t_{k+1}\right)+s\left(t_{k}\right)}{2} r_{j, k}^{\alpha}\right) \\
& =\frac{-h^{2}}{2 m}\left[\frac{\left(s_{i+1}^{j+1}-2 s_{i}^{j+1}+s_{i-1}^{j+1}\right)-\left(s_{i+1}^{j}-2 s_{i}^{j}+s_{i-1}^{j}\right)}{2(\Delta x)^{2}}\right]+u_{i}^{j}\left[\frac{s_{i}^{j+1}+s_{i}^{j}}{2}\right],
\end{aligned}
$$

where

$$
r_{j, k}^{\prime}=\left(\left(t_{j+1}-t_{k}\right)^{1-\alpha}-\left(t_{j+1}-t_{k+1}\right)^{1-\alpha}\right), \quad r_{j, k}^{\prime \prime}=\left(\left(t_{j}-t_{k}\right)^{1-\alpha}-\left(t_{j}-t_{k+1}\right)^{1-\alpha}\right) .
$$

For simplicity, let we take

$$
a_{i}^{j}=i h \frac{1}{\Gamma(2-\alpha) \Delta t}, b_{i}^{j}=\frac{-h^{2}}{4 m(\Delta x)^{2}}, c_{i}^{j}=\frac{u_{i}^{j}}{2} \text {. }
$$

Then we write (3.7) again,

$$
\begin{aligned}
& a_{i}^{j}\left(\sum_{k=0}^{j}\left(s\left(t_{k+1}\right)+s\left(t_{k}\right)\right) r_{j, k}^{\prime}-\sum_{k=0}^{j-1}\left(s\left(t_{k+1}\right)+s\left(t_{k}\right)\right) r_{j, k}^{\alpha}\right) \\
& \quad=b_{i}^{j}\left[\left(s_{i+1}^{j+1}-2 s_{i}^{j+1}+s_{i-1}^{j+1}\right)-\left(s_{i+1}^{j}-2 s_{i}^{j}+s_{i-1}^{j}\right)\right]+c_{i}^{j}\left[s_{i}^{j+1}+s_{i}^{j}\right], \\
& a_{i}^{j}\left(s_{i}^{j+1}+s_{i}^{j}\right) r_{j, k}^{\alpha}-a_{i}^{j}\left(s_{i}^{j}+s_{i}^{j-1}\right) r_{j, k}^{\prime \prime}+a_{i}^{j} \sum_{k=0}^{j-1}\left(s_{i}^{k+1}+s_{i}^{k}\right) r_{j, k}^{\alpha}-a_{i}^{j} \sum_{k=0}^{j-2}\left(s_{i}^{k+1}+s_{i}^{k}\right) r_{j, k}^{\alpha} \\
& \quad=b_{i}^{j}\left[\left(s_{i+1}^{j+1}-2 s_{i}^{j+1}+s_{i-1}^{j+1}\right)-\left(s_{i+1}^{j}-2 s_{i}^{j}+s_{i-1}^{j}\right)\right]+c_{i}^{j}\left[s_{i}^{j+1}+s_{i}^{j}\right] .
\end{aligned}
$$

Now we rearrange the equality (3.8) as below:

$$
\begin{aligned}
& \left(a_{i}^{j} r_{j, k}^{\alpha}+2 b_{i}^{j}-c_{i}^{j}\right) s_{i}^{j+1} \\
& =\left(a_{i}^{j}\left(r_{j, k}^{\prime \prime}-r_{j, k}^{\alpha}\right)-2 b_{i}^{j}+c_{i}^{j}\right) s_{i}^{j}-a_{i}^{j} \sum_{k=0}^{j-1}\left(s_{i}^{k+1}+s_{i}^{k}\right) r_{j, k}^{\alpha}+a_{i}^{j} \sum_{k=0}^{j-2}\left(s_{i}^{k+1}+s_{i}^{k}\right) r_{j, k}^{\alpha} \\
& \quad+b_{i}^{j}\left(s_{i+1}^{j+1}+s_{i-1}^{j+1}+s_{i+1}^{j}-2 s_{i}^{j}+s_{i-1}^{j}\right)+a_{i}^{j} s_{i}^{j-1} r_{j, k}^{\prime \prime} .
\end{aligned}
$$

Next, we will present the stability analysis of the numerical scheme with RL sense. 


\subsubsection{Stability analysis of the numerical scheme}

To show the efficiency of the numerical scheme with the stability analysis, we assume that $h_{i}^{j}=s_{i}^{j}-p_{i}^{j}$ where $p_{i}^{j}$ is the approximate solution of the modified equation at the given point in time and space $\left(x_{i}, t_{j}\right)$, $(i=1,2, \ldots, N, j=1,2, \ldots, M)$, also the error for approximation is given as $h^{j}=\left[h_{1}^{j}, h_{2}^{j}, \ldots, h_{N}^{j}\right]$.

The error committed while solving the Schrödinger equation with RL sense is given as:

$$
\begin{aligned}
& \left(a_{i}^{j} r_{j, k}^{\prime}+2 b_{i}^{j}-c_{i}^{j}\right) h_{i}^{j+1} \\
& =\left(a_{i}^{j}\left(r_{j, k}^{\prime \prime}-r_{j, k}^{\alpha}\right)-2 b_{i}^{j}+c_{i}^{j}\right) h_{i}^{j}-a_{i}^{j} \sum_{k=0}^{j-1}\left(h_{i}^{k+1}+h_{i}^{k}\right) r_{j, k}^{\alpha}+a_{i}^{j} \sum_{k=0}^{j-2}\left(h_{i}^{k+1}+h_{i}^{k}\right) r_{j, k}^{\alpha} \\
& \quad+b_{i}^{j}\left(h_{i+1}^{j+1}+h_{i-1}^{j+1}+h_{i+1}^{j}-2 h_{i}^{j}+h_{i-1}^{j}\right)+a_{i}^{j} h_{i}^{j-1} r_{j, k}^{\alpha} .
\end{aligned}
$$

While studying the stability, we take forms as below:

$$
h_{m}(x, t)=\exp [a t] \exp \left[i k_{m} x\right] .
$$

And,

$$
\begin{aligned}
& h_{n}^{j}=\exp [a t] \exp \left[i k_{m} x\right], \\
& h_{n}^{j+1}=\exp [a(t+\Delta t)] \exp \left[i k_{m} x\right], \\
& h_{n+1}^{j}=\exp [a t] \exp \left[i k_{m}(x+\Delta x)\right], \\
& h_{n-1}^{j}=\exp [a t] \exp \left[i k_{m}(x-\Delta x)\right] \text {, } \\
& \mathrm{h}_{\mathrm{n}+1}^{\mathrm{j}+1}=\exp [\mathrm{a}(\mathrm{t}+\Delta \mathrm{t})] \exp \left[i k_{\mathrm{m}}(\mathrm{x}+\Delta \mathrm{x})\right], \\
& h_{n-1}^{j+1}=\exp [a(t+\Delta t)] \exp \left[i k_{m}(x-\Delta x)\right], \\
& h_{n-1}^{j-1}=\exp [a(t-\Delta t)] \exp \left[i k_{m}(x-\Delta x)\right] \text {, } \\
& h_{n}^{j-1}=\exp [a(t-\Delta t)] \exp \left[i k_{m}(x)\right],
\end{aligned}
$$

where $k_{m}=\frac{\pi m}{L}, m=1,2, \ldots, M=\frac{L}{\Delta x}$. Now put the aboves in equation (3.9), then we obtain

$$
\begin{aligned}
& \left(a_{i}^{j} r_{j, k}^{\alpha}+2 b_{i}^{j}-c_{i}^{j}\right) \exp [a(t+\Delta t)] \exp \left[i k_{m} x\right] \\
& =\left(a_{i}^{j}\left(r_{j, k}^{\prime \prime}-r_{j, k}^{\alpha}\right)-2 b_{i}^{j}+c_{i}^{j}\right) \exp [a t] \exp \left[i k_{m} x\right] \\
& -a_{i}^{j} \sum_{k=0}^{j-1}\left(\left(\exp [a(t+\Delta t)] \exp \left[i k_{m} x\right]+\exp [a(\Delta t)] \exp \left[i k_{m} x\right]\right)\right) r_{j, k}^{\alpha} \\
& +a_{i}^{j} \sum_{k=0}^{j-2}\left(\left(\exp [a(t+\Delta t)] \exp \left[i k_{m} x\right]+\exp [a(\Delta t)] \exp \left[i k_{m} x\right]\right)\right) r_{j, k}^{\prime \prime} \\
& +b_{i}^{j}\left(\begin{array}{c}
\exp [a(t+\Delta t)] \exp \left[i k_{m}(x+\Delta x)\right]-\exp [a(t+\Delta t)] \exp \left[i k_{m}(x-\Delta x)\right] \\
-\exp [a t] \exp \left[i k_{m}(x+\Delta x)\right]+\exp [a t] \exp \left[i k_{m}(x-\Delta x)\right]
\end{array}\right) \\
& +a_{i}^{j} \exp [a(t-\Delta t)] \exp \left[i k_{m} \Delta x\right] r_{j, k}^{\alpha}
\end{aligned}
$$

Let do simplication with $\exp [a t] \exp \left[i k_{m} x\right]$ on both sides, then we obtain the following,

$$
\begin{aligned}
\left(a_{i}^{j} r_{j, k}^{\prime}+2 b_{i}^{j}-c_{i}^{j}\right) \exp [a(\Delta t)]= & \left(a_{i}^{j}\left(r_{j, k}^{\prime \prime}-r_{j, k}^{\alpha}\right)-2 b_{i}^{j}+c_{i}^{j}\right) \\
& -a_{i}^{j} \sum_{k=0}^{j-1}(\exp [a(\Delta t)]+1) r_{j, k}^{\prime}+a_{i}^{j} \sum_{k=0}^{j-2}(\exp [a(\Delta t)]+1) r_{j, k}^{\prime \prime}
\end{aligned}
$$




$$
+b_{i}^{j}\left(\begin{array}{c}
\exp [a(\Delta t)] \exp \left[i k_{m}(\Delta x)\right] \\
+\exp [a(\Delta t)] \exp \left[i k_{m}(-\Delta x)\right] \\
+\exp \left[i k_{m}(\Delta x)\right]+\exp \left[i k_{m}(-\Delta x)\right]
\end{array}\right)+a_{i}^{j} \exp [a(-\Delta t)] r_{j, k}^{\alpha} .
$$

Rearranging the above, we obtain

$$
\begin{aligned}
& \exp [a(\Delta t)]\left\{\begin{array}{c}
a_{i}^{j} r_{j, k}^{\prime}+2 b_{i}^{j}-c_{i}^{j}-a_{i}^{j} \cdot j\left(\begin{array}{c}
\prime \prime \\
r_{j, k}^{\alpha}-r_{j, k}^{\alpha}
\end{array}\right) \\
-b_{i}^{j}\left(\exp \left[i k_{m}(\Delta x)\right]+\exp \left[i k_{m}(-\Delta x)\right]\right)
\end{array}\right\} \\
& =\left\{\begin{array}{c}
a_{i}^{j}\left(\begin{array}{c}
\prime \prime \\
r_{j, k}^{\alpha}-r_{j, k}^{\alpha}
\end{array}\right)-2 b_{i}^{j}+c_{i}^{j}+a_{i}^{j} \cdot j\left(r_{j, k}^{\prime \prime}-r_{j, k}^{\alpha}\right. \\
+b_{i}^{j}\left(\exp \left[i k_{m}(\Delta x)\right]+\exp \left[i k_{m}(-\Delta x)\right]\right)+a_{i}^{j} \exp [a(-\Delta t)] r_{j, k}^{\alpha}
\end{array}\right\} .
\end{aligned}
$$

Then

$$
\exp [a(\Delta t)]=\frac{\left\{\begin{array}{c}
a_{i}^{j}\left(r_{j, k}^{\prime \prime}-r_{j, k}^{\alpha}\right)-2 b_{i}^{j}+c_{i}^{j}+a_{i}^{j} \cdot j\left(\begin{array}{c}
\prime \prime \\
r_{j, k}^{\alpha}-r_{j, k}^{\alpha}
\end{array}\right) \\
+b_{i}^{j}\left(\exp \left[i k_{m}(\Delta x)\right]+\exp \left[i k_{m}(-\Delta x)\right]\right)+a_{i}^{j} \exp [a(-\Delta t)] r_{j, k}^{\alpha}
\end{array}\right\}}{\left\{a_{i}^{j} r_{j, k}^{\prime}+2 b_{i}^{j}-c_{i}^{j}-a_{i}^{j} \cdot j\left(r_{j, k}^{\prime \prime}-r_{j, k}^{\alpha}\right)-b_{i}^{j}\left(\exp \left[i k_{m}(\Delta x)\right]+\exp \left[i k_{m}(-\Delta x)\right]\right)\right\}} .
$$

Note that the condition for stability analyis is given by the following inequality

$$
\frac{h_{i}^{j+1}}{h_{i}^{j}}=\exp [a(\Delta t)] \text {. }
$$

Thus if

$$
\left|\frac{h_{i}^{j+1}}{h_{i}^{j}}\right| \leqslant 1
$$

from equation (3.10) we have the following

$$
\left|\frac{h_{i}^{j+1}}{h_{i}^{j}}\right|=\exp [a(\Delta t)]|=| \frac{\left\{\begin{array}{c}
a_{i}^{j}\left(\begin{array}{c}
\prime \prime \\
r_{j, k}^{\alpha}-r_{j, k}^{\alpha}
\end{array}\right)-2 b_{i}^{j}+c_{i}^{j}+a_{i}^{j} \cdot j\left(\begin{array}{c}
\prime \prime \\
r_{j, k}^{\alpha}-r_{j, k}^{\alpha}
\end{array}\right) \\
+b_{i}^{j}\left(\exp \left[i k_{m}(\Delta x)\right]+\exp \left[i k_{m}(-\Delta x)\right]\right)+a_{i}^{j} \exp [a(-\Delta t)] r_{j, k}^{\alpha}
\end{array}\right\}}{\left\{\begin{array}{c}
a_{i}^{j}{ }_{j, k}^{\alpha}+2 b_{i}^{j}-c_{i}^{j}-a_{i}^{j} \cdot j\left(r_{j, k}^{\prime \prime}-r_{j, k}^{\alpha}\right) \\
-b_{i}^{j}\left(\exp \left[i k_{m}(\Delta x)\right]+\exp \left[i k_{m}(-\Delta x)\right]\right)
\end{array}\right\}} \mid
$$

Then the condition for stability is given as

$$
\left|\left\{\begin{array}{c}
a_{i}^{j}\left(\begin{array}{c}
\prime \prime \\
r_{j, k}^{\alpha}-r_{j, k}^{\alpha}
\end{array}\right)-2 b_{i}^{j}+c_{i}^{j}+a_{i}^{j} \cdot j\left(\begin{array}{c}
\prime \prime \\
r_{j, k}^{\alpha}-r_{j, k}^{\alpha}
\end{array}\right) \\
+b_{i}^{j}\left(\exp \left[i k_{m}(\Delta x)\right]+\exp \left[i k_{m}(-\Delta x)\right]+a_{i}^{j} \exp [a(-\Delta t)] r_{j, k}^{\alpha}\right.
\end{array}\right\}\right| \leqslant 1 .
$$


Thus from above statement we can present the following theorem.

Theorem 3.2. The Crank-Nicholson scheme for solving the fractional Schrödinger equation with RL sense is stable if inequality (3.11) is satisfied.

\subsection{Atangana-Baleanu derivative with Riemann-Liouville approach and stability analysis of numerical scheme}

The time dependent Schrödinger equation with ABR (Atangana-Baleanu derivative with RiemannLiouville sense) operator is given by

$$
i h{ }_{0}^{A B R} D_{t}^{\alpha}[s(x, t)]=\frac{-h^{2}}{2 m} \frac{\partial^{2} s(x, t)}{\partial x^{2}}+u(x) s(x, t), 0 \leqslant \alpha \leqslant 1 .
$$

The numerical approximation ABR derivative is given below.

$$
{ }_{0}^{A B R} D_{t}^{\alpha}[f(t)]=\frac{B(\alpha)}{1-\alpha} \frac{d}{d t} \int_{a}^{t} f(y) E_{\alpha}\left[-\frac{\alpha}{1-\alpha}(t-y)^{\alpha}\right] d y, 0 \leqslant \alpha \leqslant 1 .
$$

If we do same steps like numerical approximation of CFR and RL, we will obtain below:

$$
{ }_{0}^{A B R} D_{t}^{\alpha}[f(t)]=\frac{B(\alpha)}{\Gamma(1-\alpha) \Delta t}\left\{\sum_{k=0}^{j} \frac{f\left(t_{k+1}\right)+f\left(t_{k}\right)}{2} \theta_{j}^{\alpha, 1}-\sum_{k=0}^{j-1} \frac{f\left(t_{k+1}\right)+f\left(t_{k}\right)}{2} \beta_{j}^{\alpha, 1}\right\}+A_{\alpha, j, k}
$$

where

$$
\begin{aligned}
& \theta_{j}^{\alpha, 1}=\left(t_{j+1}-t_{k+1}\right) E_{\alpha, 2}\left[-\frac{\alpha}{1-\alpha}\left(t_{j+1}-t_{k+1}\right)^{\alpha}\right]+\left(t_{j+1}-t_{k}\right) E_{\alpha, 2}\left[-\frac{\alpha}{1-\alpha}\left(t_{j+1}-t_{k}\right)^{\alpha}\right], \\
& \beta_{j}^{\alpha, 1}=\left(t_{j}-t_{k+1}\right) E_{\alpha, 2}\left[-\frac{\alpha}{1-\alpha}\left(t_{j}-t_{k+1}\right)^{\alpha}\right]+\left(t_{j}-t_{k}\right) E_{\alpha, 2}\left[-\frac{\alpha}{1-\alpha}\left(t_{j}-t_{k}\right)^{\alpha}\right],
\end{aligned}
$$

and $A_{\alpha, j, k}$ is the remainder term given by equality below

$$
A_{\alpha, j, k}=\frac{B(\alpha)}{\Gamma(1-\alpha) \Delta t}\left\{\begin{array}{l}
\sum_{k=0}^{j} \int_{t_{k}}^{t_{k+1}}\left\{f(y)-f\left(t_{k+1}\right)\right\} E_{\alpha}\left[-\frac{\alpha}{1-\alpha}\left(t_{j+1}-y\right)^{\alpha}\right] d y \\
-\sum_{k=0}^{j-1} \int_{t_{k}}^{t_{k+1}}\left\{f(y)-f\left(t_{k+1}\right)\right\} E_{\alpha}\left[-\frac{\alpha}{1-\alpha}\left(t_{j}-y\right)^{\alpha}\right] d y
\end{array}\right\}
$$

Now we will consider the Schrödinger equation with ABR approximation.

$$
\text { ih } \begin{aligned}
& \frac{B(\alpha)}{\Gamma(1-\alpha) \Delta t}\left(\sum_{k=0}^{j} \frac{s\left(t_{k+1}\right)+s\left(t_{k}\right)}{2} \theta_{j}^{\alpha, 1}-\sum_{k=0}^{j-1} \frac{s\left(t_{k+1}\right)+s\left(t_{k}\right)}{2} \beta_{j}^{\alpha, 1}\right) \\
\quad= & \frac{-h^{2}}{2 m}\left[\frac{\left(s_{i+1}^{j+1}-2 s_{i}^{j+1}+s_{i-1}^{j+1}\right)-\left(s_{i+1}^{j}-2 s_{i}^{j}+s_{i-1}^{j}\right)}{2(\Delta x)^{2}}\right]+u_{i}^{j}\left[\frac{s_{i}^{j+1}+s_{i}^{j}}{2}\right] .
\end{aligned}
$$

For simplicity, let we take

$$
v_{i}^{j}=i h \frac{B(\alpha)}{\Gamma(1-\alpha) \Delta t}, y_{i}^{j}=\frac{-h^{2}}{4 m(\Delta x)^{2}}, z_{i}^{j}=\frac{u_{i}^{j}}{2} .
$$

Then we write (3.12) again,

$$
\begin{aligned}
& v_{i}^{j}\left(\sum_{k=0}^{j}\left(s\left(t_{k+1}\right)+s\left(t_{k}\right)\right) \theta_{j}^{\alpha, 1}-\sum_{k=0}^{j-1}\left(s\left(t_{k+1}\right)+s\left(t_{k}\right)\right) \beta_{j}^{\alpha, 1}\right) \\
& \quad=y_{i}^{j}\left[\left(s_{i+1}^{j+1}-2 s_{i}^{j+1}+s_{i-1}^{j+1}\right)-\left(s_{i+1}^{j}-2 s_{i}^{j}+s_{i-1}^{j}\right)\right]+z_{i}^{j}\left[s_{i}^{j+1}+s_{i}^{j}\right],
\end{aligned}
$$




$$
\begin{aligned}
& v_{i}^{j}\left(s_{i}^{j+1}+s_{i}^{j}\right) \theta_{j}^{\alpha, 1}-v_{i}^{j}\left(s_{i}^{j}+s_{i}^{j-1}\right) \beta_{j}^{\alpha, 1}+v_{i}^{j} \sum_{k=0}^{j-1}\left(s_{i}^{k+1}+s_{i}^{k}\right) \theta_{j}^{\alpha, 1}-v_{i}^{j} \sum_{k=0}^{j-2}\left(s_{i}^{k+1}+s_{i}^{k}\right) \beta_{j}^{\alpha, 1} \\
& \quad=y_{i}^{j}\left[\left(s_{i+1}^{j+1}-2 s_{i}^{j+1}+s_{i-1}^{j+1}\right)-\left(s_{i+1}^{j}-2 s_{i}^{j}+s_{i-1}^{j}\right)\right]+z_{i}^{j}\left[s_{i}^{j+1}+s_{i}^{j}\right] .
\end{aligned}
$$

Now we rearrange the equality (3.13) as below:

$$
\begin{aligned}
& \left(v_{i}^{j} \theta_{j}^{\alpha, 1}+2 y_{i}^{j}-z_{i}^{j}\right) s_{i}^{j+1} \\
& =\left(v_{i}^{j}\left(\beta_{j}^{\alpha, 1}-\theta_{j}^{\alpha, 1}\right)-2 y_{i}^{j}+z_{i}^{j}\right) s_{i}^{j}-v_{i}^{j} \sum_{k=0}^{j-1}\left(s_{i}^{k+1}+s_{i}^{k}\right) \theta_{j}^{\alpha, 1}+v_{i}^{j} \sum_{k=0}^{j-2}\left(s_{i}^{k+1}+s_{i}^{k}\right) \beta_{j}^{\alpha, 1} \\
& \quad+v_{i}^{j}\left(s_{i+1}^{j+1}+s_{i-1}^{j+1}+s_{i+1}^{j}-2 s_{i}^{j}+s_{i-1}^{j}\right)+v_{i}^{j} s_{i}^{j-1} \beta_{j}^{\alpha, 1} .
\end{aligned}
$$

Next, we will present the stability analysis of the numerical scheme with ABR sense.

\subsubsection{Stability analysis of the numerical scheme}

To show the efficiency of the numerical scheme with the stability analysis, we assume that $l_{i}^{j}=s_{i}^{j}-p_{i}^{j}$ where $p_{i}^{j}$ is the approximate solution of the modified equation at the given point in time and space $\left(x_{i}, t_{j}\right)$, $(i=1,2, \ldots, N, j=1,2, \ldots, M)$, also the error for approximation is given as $l^{j}=\left[l_{1}^{j}, l_{2}^{j}, \ldots, l_{N}^{j}\right]$.

The error committed while solving the Schrödinger equation with CFR is given as:

$$
\begin{aligned}
& \left(v_{i}^{j} \theta_{j}^{\alpha, 1}+2 y_{i}^{j}-z_{i}^{j}\right) l_{i}^{j+1} \\
& =\left(v_{i}^{j}\left(\beta_{j}^{\alpha, 1}-\theta_{j}^{\alpha, 1}\right)-2 y_{i}^{j}+z_{i}^{j}\right) l_{i}^{j}-v_{i}^{j} \sum_{k=0}^{j-1}\left(l_{i}^{k+1}+l_{i}^{k}\right) \theta_{j}^{\alpha, 1}+v_{i}^{j} \sum_{k=0}^{j-2}\left(l_{i}^{k+1}+l_{i}^{k}\right) \beta_{j}^{\alpha, 1} \\
& \quad+v_{i}^{j}\left(l_{i+1}^{j+1}+l_{i-1}^{j+1}+l_{i+1}^{j}-2 l_{i}^{j}+l_{i-1}^{j}\right)+v_{i}^{j} l_{i}^{j-1} \beta_{j}^{\alpha, 1} .
\end{aligned}
$$

While studying the stability, we take forms as below:

$$
l_{m}(x, t)=\exp [a t] \exp \left[i k_{m} x\right]
$$

And,

$$
\begin{aligned}
& l_{n}^{j}=\exp [a t] \exp \left[i k_{m} x\right], \\
& l_{n+1}^{j}=\exp [a t] \exp \left[i k_{m}(x+\Delta x)\right], \\
& l_{n}^{j+1}=\exp [a(t+\Delta t)] \exp \left[i k_{m} x\right], \\
& l_{n+1}^{j+1}=\exp [a(t+\Delta t)] \exp \left[i k_{m}(x+\Delta x)\right], \\
& l_{n-1}^{j}=\exp [a t] \exp \left[i k_{m}(x-\Delta x)\right], \\
& l_{n-1}^{j-1}=\exp [a(t-\Delta t)] \exp \left[i k_{m}(x-\Delta x)\right] \text {, } \\
& l_{n-1}^{j+1}=\exp [a(t+\Delta t)] \exp \left[i k_{m}(x-\Delta x)\right], \\
& l_{n}^{j-1}=\exp [a(t-\Delta t)] \exp \left[i k_{m}(x)\right],
\end{aligned}
$$

where $k_{m}=\frac{\pi m}{L}, m=1,2, \ldots, M=\frac{L}{\Delta x}$. Now put the aboves in equation (3.14), then we obtain

$$
\begin{aligned}
& \left(v_{i}^{j} \theta_{j}^{\alpha, 1}+2 y_{i}^{j}-z_{i}^{j}\right) \exp [a(t+\Delta t)] \exp \left[i k_{m} x\right] \\
& =\left(v_{i}^{j}\left(\beta_{j}^{\alpha, 1}-\theta_{j}^{\alpha, 1}\right)-2 y_{i}^{j}+z_{i}^{j}\right) \exp [a t] \exp \left[i k_{m} x\right] \\
& \quad-v_{i}^{j} \sum_{k=0}^{j-1}\left(\exp [a(t+\Delta t)] \exp \left[i k_{m} x\right]+\exp [a(\Delta t)] \exp \left[i k_{m} x\right]\right) \theta_{j}^{\alpha, 1} \\
& \quad+v_{i}^{j} \sum_{k=0}^{j-2}\left(\exp [a(t+\Delta t)] \exp \left[i k_{m} x\right]+\exp [a(\Delta t)] \exp \left[i k_{m} x\right]\right) \beta_{j}^{\alpha, 1}
\end{aligned}
$$




$$
\begin{aligned}
& +y_{i}^{j}\left(\begin{array}{c}
\exp [a(t+\Delta t)] \exp \left[i k_{m}(x+\Delta x)\right]-\exp [a(t+\Delta t)] \exp \left[i k_{m}(x-\Delta x)\right] \\
-\exp [a t] \exp \left[i k_{m}(x+\Delta x)\right]+\exp [a t] \exp \left[i k_{m}(x-\Delta x)\right]
\end{array}\right) \\
& +z_{i}^{j} \exp [a(t-\Delta t)] \exp \left[i k_{m} \Delta x\right] \beta_{j}^{\alpha, 1} .
\end{aligned}
$$

Let do simplication with $\exp [a t] \exp \left[i k_{m} x\right]$ on both sides, then we obtain the following,

$$
\begin{aligned}
\left(v_{i}^{j} \theta_{j}^{\alpha, 1}+2 y_{i}^{j}-z_{i}^{j}\right) \exp [a(\Delta t)]= & \left(v_{i}^{j}\left(\theta_{j}^{\alpha, 1}-\beta_{j}^{\alpha, 1}\right)-2 y_{i}^{j}+z_{i}^{j}\right)-v_{i}^{j} \sum_{k=0}^{j-1}(\exp [a(\Delta t)]+1) \theta_{j}^{\alpha, 1} \\
& +v_{i}^{j} \sum_{k=0}^{j-2}(\exp [a(\Delta t)]+1) \beta_{j}^{\alpha, 1} \\
& +y_{i}^{j}\left(\begin{array}{c}
\exp [a(\Delta t)] \exp \left[i k_{m}(\Delta x)\right] \\
+\exp [a(\Delta t)] \exp \left[i k_{m}(-\Delta x)\right] \\
+\exp \left[i k_{m}(\Delta x)\right]+\exp \left[i k_{m}(-\Delta x)\right]
\end{array}\right)+v_{i}^{j} \exp [a(-\Delta t)] \beta_{j}^{\alpha, 1} .
\end{aligned}
$$

Rearranging the above, we obtain

$$
\begin{aligned}
& \exp [a(\Delta t)]\left\{v_{i}^{j} \theta_{j}^{\alpha, 1}+2 y_{i}^{j}-z_{i}^{j}-v_{i}^{j} \cdot j\left(\beta_{j}^{\alpha, 1}-\theta_{j}^{\alpha, 1}\right)-y_{i}^{j}\left(\exp \left[i k_{m}(\Delta x)\right]+\exp \left[i k_{m}(-\Delta x)\right]\right)\right\} \\
& \quad=\left\{\begin{array}{c}
v_{i}^{j}\left(\beta_{j}^{\alpha, 1}-\theta_{j}^{\alpha, 1}\right)-2 y_{i}^{j}+z_{i}^{j}+v_{i}^{j} \cdot j\left(\beta_{j}^{\alpha, 1}-\theta_{j}^{\alpha, 1}\right) \\
+y_{i}^{j}\left(\exp \left[i k_{m}(\Delta x)\right]+\exp \left[i k_{m}(-\Delta x)\right]\right)+v_{i}^{j} \exp [a(-\Delta t)] \beta_{j}^{\alpha, 1}
\end{array}\right\} .
\end{aligned}
$$

Then

$$
\exp [a(\Delta t)]=\frac{\left\{\begin{array}{c}
v_{i}^{j}\left(\beta_{j}^{\alpha, 1}-\theta_{j}^{\alpha, 1}\right)-2 y_{i}^{j}+z_{i}^{j}+v_{i}^{j} \cdot j\left(\beta_{j}^{\alpha, 1}-\theta_{j}^{\alpha, 1}\right) \\
+y_{i}^{j}\left(\exp \left[i k_{m}(\Delta x)\right]+\exp \left[i k_{m}(-\Delta x)\right]\right)+v_{i}^{j} \exp [a(-\Delta t)] \beta_{j}^{\alpha, 1}
\end{array}\right\}}{\left\{v_{i}^{j} \theta_{j}^{\alpha, 1}+2 y_{i}^{j}-z_{i}^{j}-v_{i}^{j} \cdot j\left(\beta_{j}^{\alpha, 1}-\theta_{j}^{\alpha, 1}\right)-y_{i}^{j}\left(\exp \left[i k_{m}(\Delta x)\right]+\exp \left[i k_{m}(-\Delta x)\right]\right)\right\}} .
$$

Note that the condition for stability analysis is given by the following inequality

$$
\frac{l_{i}^{j+1}}{l_{i}^{j}}=\exp [a(\Delta t)] \text {. }
$$

Thus if

$$
\left|\frac{l_{i}^{j+1}}{l_{i}^{j}}\right| \leqslant 1,
$$

from equation (3.15) we have the following

$$
\left|\frac{l_{i}^{j+1}}{l_{i}^{j}}\right|=|\exp [a(\Delta t)]|=\left|\frac{\left\{\begin{array}{c}
v_{i}^{j}\left(\beta_{j}^{\alpha, 1}-\theta_{j}^{\alpha, 1}\right)-2 y_{i}^{j}+z_{i}^{j}+v_{i}^{j} \cdot j\left(\beta_{j}^{\alpha, 1}-\theta_{j}^{\alpha, 1}\right) \\
+y_{i}^{j}\left(\exp \left[i k_{m}(\Delta x)\right]+\exp \left[i k_{m}(-\Delta x)\right]+v_{i}^{j} \exp [a(-\Delta t)] \beta_{j}^{\alpha, 1}\right.
\end{array}\right\}}{\left\{\begin{array}{c}
v_{i}^{j} \theta_{j}^{\alpha, 1}+2 y_{i}^{j}-z_{i}^{j}-v_{i}^{j} \cdot j\left(\beta_{j}^{\alpha, 1}-\theta_{j}^{\alpha, 1}\right) \\
-y_{i}^{j}\left(\exp \left[i k_{m}(\Delta x)\right]+\exp \left[i k_{m}(-\Delta x)\right]\right)
\end{array}\right\}}\right| .
$$

Then the condition for stability is given as

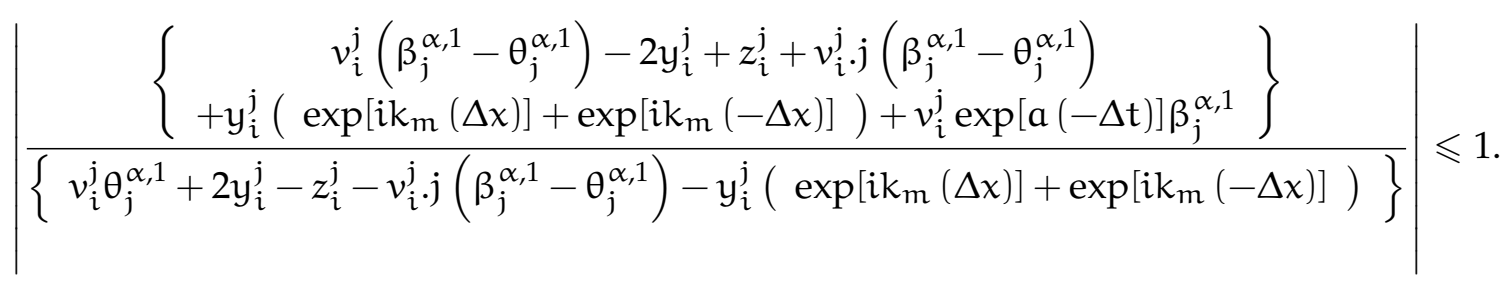

Thus from that above statement we can present the following theorem. 
Theorem 3.3. The Crank-Nicholson scheme for solving the fractional Schrödinger equation with ABR is stable if inequality (3.16) is satisfied.

\section{Conclusion}

A new numerical scheme based on Riemann-Liouville, Caputo-Fabrizio and Atangana-Baleanu fractional derivatives in Riemann-Liouville sense are presented in this paper. The three numerical approximations are used to solve the Schrödinger equation with the three derivatives based on power law, exponential decay law and generalized Mittag-Leffler law. We studied in detail the stability and the convergence of the used numerical scheme for solving the modified models. Within some conditions, the numerical scheme suggested here is stable and convergent.

\section{Acknowledgment}

The authors extend their sincere appreciations to the Deanship of Science Research at King Saud University for funding this prolific research group PRG-1437-35.

\section{References}

[1] O. J. J. Algahtani, Comparing the Atangana-Baleanu and Caputo-Fabrizio derivative with fractional order: Allen Cahn model, Chaos Solitons Fractals, 89 (2016), 552-559. 1

[2] T. B. S. Alkahtani, Chua's circuit model with AtanganaBaleanu derivative with fractional order, Chaos Solitons Fractals, 89 (2016), 547-551. 1

[3] A. Atangana, On the new fractional derivative and application to nonlinear Fisher's reaction-diffusion equation, Appl. Math. Comput., 273 (2016), 948-956. 1

[4] A. Atangana, D. Baleanu, New fractional derivatives with nonlocal and non-singular kernel: theory and application to heat transfer model, Therm. Sci., 20 (2016), 763-769. 1, 2.2

[5] A. Atangana, J. F. Gomez-Aquilar, Numerical approximation of Riemann-Liouville definition of fractional derivative: From Riemann- Liouville to Atangana-Baleanu, Appl. Numer. Math., (2016), submitted. 3

[6] A. Atangana, I. Koca, Chaos in a simple nonlinear system with Atangana-Baleanu derivatives with fractional order, Chaos Solitons Fractals, 89 (2016), 447-454. 1

[7] H. Bulut, F. B. M. Belgacem, H. M. Baskonus, Some new analytical solutions for the nonlinear Time-Fractional KdVBurgers-Kuramoto equation, Adv. Math. Stat. Sci., 2 (2015), 118-129. 1

[8] M. Caputo, M. Fabrizio, A new definition of fractional derivative without singular kernel, Progr. Fract. Differ. Appl., 1 (2015), 73-85. 1

[9] M. Caputo, M. Fabrizio, Applications of new time and spatial fractional derivatives with exponential kernels, Progr. Fract. Differ. Appl., 2 (2016), 1-11. 1

[10] J. F. Gómez-Aguilar, M. Guía-Calderón, J. Rosales-García, J. Bernal-Alvarado, Analysis of equivalent circuits for cells: a fractional calculus approach, Ing. Investig. Tecnolog., UNAM, 13 (2012), 375-384. 1

[11] J. F. Gómez-Aguilar, R. Razo-Hernández, D. Granados-Lieberman, A physical interpretation of fractional calculus in observables terms: analysis of the fractional time constant and the transitory response, Rev. Mexicana Fís., 60 (2014), 32-38. 1

[12] J. Losada, J. J. Nieto, Properties of a new fractional derivative without singular kernel, Progr. Fract. Differ. Appl., 1 (2015), 87-92. 1

[13] S. G. Samko, A. A. Kilbas, O. I. Marichev, Fractional integrals and derivatives, Theory and applications, Edited and with a foreword by S. M. Nikol'skiĭ, Translated from the 1987 Russian original, Revised by the authors, Gordon and Breach Science Publishers, Yverdon, (1993). 2.1

[14] X.-J. Yang, Fractional derivatives of constant and variable orders applied to anomalous relaxation models in heat-transfer problems, Therm. Sci., 21 (2016), 1161-1171. 3.1

[15] X.-J. Yang, H. M. Srivastava, J. A. Tenreiro Machado, A new fractional derivative without singular kernel: application to the modelling of the steady heat flow, Therm. Sci., 20 (2016), 753-756. 2.3

[16] X.-J. Yang, J. A. Tenreiro Machado, A new fractional operator of variable order: application in the description of anomalous diffusion, Phys. A, 481 (2017), 276-283. 3.1

[17] X.-J. Yang, J. A. Tenreiro Machado, J. Hristov, Nonlinear dynamics for local fractional Burgers' equation arising in fractal flow, Nonlinear Dynam., 84 (2016), 3-7. 1

[18] X.-J. Yang, J. A. Tenreiro Machado, H. M. Srivastava, A new numerical technique for solving the local fractional diffusion equation: two-dimensional extended differential transform approach, Appl. Math. Comput., 274 (2016), 143-151. 1 